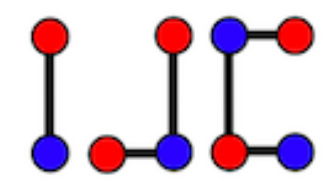

\title{
On the Ramsey number of 4-cycle versus wheel
}

\author{
Enik Noviani, Edy Tri Baskoro \\ Combinatorial Mathematics Research Group \\ Faculty of Mathematics and Natural Sciences, Institut Teknologi Bandung \\ Bandung, Indonesia \\ eniknoviani@students.itb.ac.id, ebaskoro@math.itb.ac.id
}

\begin{abstract}
For any fixed graphs $G$ and $H$, the Ramsey number $R(G, H)$ is the smallest positive integer $n$ such that for every graph $F$ on $n$ vertices must contain $G$ or the complement of $F$ contains $H$. The girth of graph $G$ is a length of the shortest cycle. A $k$-regular graph with the girth $g$ is called a $(k, g)$-graph. If the number of vertices in $(k, g)$-graph is minimized then we call this graph a $(k, g)$-cage. In this paper, we derive the bounds of Ramsey number $R\left(C_{4}, W_{n}\right)$ for some values of $n$. By modifying $(k, 5)$-graphs, for $k=7$ or 9 , we construct these corresponding $\left(C_{4}, W_{n}\right)$-good graphs.
\end{abstract}

Keywords: Ramsey number, good graph, order, cycle, wheel, girth Mathematics Subject Classification: 05C55

\section{Introduction}

In this paper, we consider a finite undirected graphs without loops or multiple edges. Let $G$ be graphs. The sets of vertices and edges of graph $G$ are denoted by $V(G)$ and $E(G)$, respectively. The symbols $\delta(G)$ and $\triangle(G)$ represents the smallest and the greatest degree of vertices in $G$, respectively. Let $C_{n}$ be a cycle with $n$ vertices and $W_{n}$ be a wheel on $n$ vertices obtained from a $C_{n-1}$ by adding one vertex $x$ and making $x$ adjacent to all vertices of the $C_{n-1}$. The girth of a graph $G$ is the length of its shortest cycle in $G$. A $k$-regular graph with girth $g$ is called a $(k, g)$-graph. A $(k, g)$-graph with minimum number of vertices is called a $(k, g)$-cage. For fixed graphs $G$ and $H$, a graph $F$ is called a $(G, H)$-good graph if $F$ contains no $G$ and $F$ complement contains no

Received: 9 July 2015, Revised: 26 August 2016, Accepted: 14 September 2016. 
$H$. Any $(G, H)$-good graph with $n$ vertices will be called a $(G, H, n)$-good graph. The Ramsey number $R(G, H)$ is the smallest positive integer $n$ such that for every graph $F$ of order $n$ contains $G$ or the complement of $F$ contain $H$. So, the Ramsey number $R(G, H)$ is the smallest positive integer $n$ such that there exists no $(G, H, n)$-good graph.

It is known that $R\left(C_{4}, W_{4}\right)=10, R\left(C_{4}, W_{5}\right)=9$ and $R\left(C_{4}, W_{6}\right)=10$ (cf.[2]). Tse [2] determined the value of $R\left(C_{4}, W_{m}\right)$ for $7 \leq m \leq 13$. Dybizbański dan Dzido [2] determined that $R\left(C_{4}, W_{m}\right)=m+4$ for $14 \leq m \leq 16$ and $R\left(C_{4}, W_{q^{2}+1}\right)=q^{2}+q+1$ for prime power $q \geq 4$. Recently, Zhang, Broersma and Chen [2] show that $R\left(C_{4}, W_{n}\right)=R\left(C_{4}, S_{n}\right)$ for $n \geq 7$. Based on this result and Parsons' results on $R\left(C_{4}, S_{n}\right)$, they derived the best possible general upper bound for $R\left(C_{4}, W_{n}\right)$ and determined some exact values of them. In general, the exact value of the Ramsey number $R\left(C_{4}, W_{n}\right)$ is still open for $n \geq 17$ with the exception for several values of $n$. In this paper, we derive the bounds of Ramsey number $R\left(C_{4}, W_{n}\right)$ for some values of $n$. By modifying $(k, 5)$-graphs, for $k=7$ or 9 , we construct these corresponding $\left(C_{4}, W_{n}\right)$-good graphs.

Theorem 1.1. Each of the following statements must hold.

(i) For any $m \geq 18$ there exists a graph $G$ of order $m$ with $\delta(G)=4$ and $G \nsupseteq C_{4}$.

(ii) For any even $m \geq 50$ there exists a graph $G$ of order $m$ with $\delta(G)=5$ and $G \nsupseteq C_{4}$.

(iii) $R\left(C_{4}, W_{2 k+1}\right) \geq R\left(C_{4}, W_{2 k}\right)$ for any $k \geq 25$.

(iv) $R\left(C_{4}, W_{m+n}\right) \geq \max \left\{R\left(C_{4}, W_{m}\right), R\left(C_{4}, W_{n}\right)\right\}$ with $\min \{m, n\} \geq 7$ and $\max \{m, n\} \geq$ 50.

Theorem 1.2. The upper and lower bounds of the Ramsey number $R\left(C_{4}, W_{m}\right)$ for any $m \in[46,93]$ are as follows.

(i) $m+6 \leq R\left(C_{4}, W_{m}\right) \leq m+7$, for $46 \leq m \leq 51$.

(ii) $m+8 \leq R\left(C_{4}, W_{m}\right) \leq m+9$, for $79 \leq m \leq 82$,

(iii) $m+8 \leq R\left(C_{4}, W_{m}\right) \leq m+10$, for $83 \leq m \leq 87$.

(iv) $97 \leq R\left(C_{4}, W_{88}\right) \leq 98$ and $m+8 \leq R\left(C_{4}, W_{m}\right) \leq m+10$, for $89 \leq m \leq 93$.

\section{Proofs of the main results}

To prove Theorems 1.1 and 1.2, we need the following two lemmas and one theorem.

Lemma 2.1. [2] If $G$ is $a\left(C_{4}, W_{m}, n\right)$-good graph for $7 \leq m \leq n-4$ then $\delta(G) \geq n-m+1$.

Lemma 2.2. [2] If $G$ contains no $C_{4}$ with $n$ vertices and $\delta(G)=d$ then $d^{2}-d+1 \leq n$.

Theorem 2.1. [2] For all integers $m \geq 11, R\left(C_{4}, W_{m}\right) \leq m+\lfloor\sqrt{m-2}\rfloor+1$.

Proof Theorem 1.1.

(i) For any integer $m \geq 18$, construct a graph $G$ on $m$ vertices with $\delta(G)=4$ and $G \nsupseteq C_{4}$ by considering the following two cases.

(a) Case $1 m=2 k, k \geq 9$.

First, if $k \neq 12$ define the vertex-set and edge-set of $G$ as follows. 
- $V(G)=\left\{a_{1}, a_{2}, \ldots, a_{k}, b_{1}, b_{2}, \ldots, b_{k}\right\}$, and

- $E(G)=\left\{a_{i} b_{i}, b_{i} a_{i+1}, a_{i} a_{i+1}, b_{i} b_{i+3}: 1 \leq i \leq k\right.$ and all indices are in $\left.\bmod k\right\}$.

Note that all indices are calculated in $\bmod k$. It is clear that vertex $a_{i}$ is adjacent to each of $\left\{b_{i}, b_{i-1}, a_{i+1}, a_{i-1}\right\}$ and $b_{i}$ is adjacent to each of $\left\{a_{i}, a_{i+1}, b_{i+3}, b_{i-3}\right\}$ for all $i=1,2, \cdots, k$. Thus, $\delta(G)=4$. Now, we will show that $G \nsupseteq C_{4}$. For a contradiction, suppose $G$ contains a $C_{4}$. Since $k \neq 12$, the four vertices of $C_{4}$ cannot be all $b_{i}$. Therefore, this $C_{4}$ must contain at least one vertex $a_{i}$. Now, consider the following 3 subcases.

- Subcase 1. $a_{i} b_{i} \in C_{4}$ for some $i$. If $a_{i}$ and $b_{i}$ are the first and second vertices of this $C_{4}$ then the possible third and fourth vertices are listed in Table 1. However, we have that no vertex 4 is adjacent to vertex 1 . Therefore, there is no such $C_{4}$ occur. Thus, $a_{i} b_{i}$ is not an edge in $C_{4}$.

\begin{tabular}{|l|l|l|l|}
\hline vertex 1 & vertex 2 & vertex 3 & vertex 4 \\
\hline$a_{i}$ & $b_{i}$ & $a_{i+1}$ & $b_{i+1}$ \\
\hline & & & $a_{i}$ \\
\hline & & & $a_{i+2}$ \\
\hline & & $b_{i+3}$ & $a_{i+3}$ \\
\hline & & & $a_{i+4}$ \\
\hline & & & $b_{i+6}$ \\
\hline & & $b_{i-3}$ & $a_{i-3}$ \\
\hline & & & $a_{i-2}$ \\
\hline & & & $a_{i-6}$ \\
\hline
\end{tabular}

Table 1. List of possible vertices of a $C_{4}$ in Subcase 1.

- Subcase 2. $b_{i} a_{i+1} \in C_{4}$ for some $i$. If $b_{i}$ and $a_{i+1}$ are the first and second vertices in this $C_{4}$, and no edge $a_{i} b_{i} \in C_{4}$, for each $i$, then the possible third and fourth vertices are presented in Table 2. Clearly, each of the possible fourth vertices is not adjacent to vertex 1 . Therefore, no $C_{4}$ is formed in this case.

\begin{tabular}{|l|l|l|l|}
\hline vertex 1 & vertex 2 & vertex 3 & vertex 4 \\
\hline$b_{i}$ & $a_{i+1}$ & $a_{i+2}$ & $b_{i+1}$ \\
\hline & & & $a_{i+3}$ \\
\hline & & $a_{i}$ & $b_{i-1}$ \\
\hline & & & $a_{i-1}$ \\
\hline
\end{tabular}

Table 2. List of possible vertices of a $C_{4}$ in Subcase 2.

- Subcase 3. $a_{i} a_{i+1}$ or $b_{i} b_{i+3} \in C_{4}$ for some $i$. From the previous subcases, we know that the edges $a_{i} b_{i}$ or $b_{i} a_{i+1}$ cannot be in this $C_{4}$. So, this $C_{4}$ only consist of edges $a_{i} a_{i+1}$ and/or $b_{i} b_{i+3}$ for some $i$. Since $k \neq 12$ then no $C_{4}$ occurs in this case. 
Therefore, if $m=2 k, k \geq 9$ and $k \neq 12$ then the above graph $G$ has $m$ vertices with $\delta(G)=4$ and $G \nsupseteq C_{4}$.

Second, for $k=12$, consider graph $G$ of order 24 in Figure 1. It can be verified that $G$ containing no $C_{4}$ and $\delta(G)=4$.

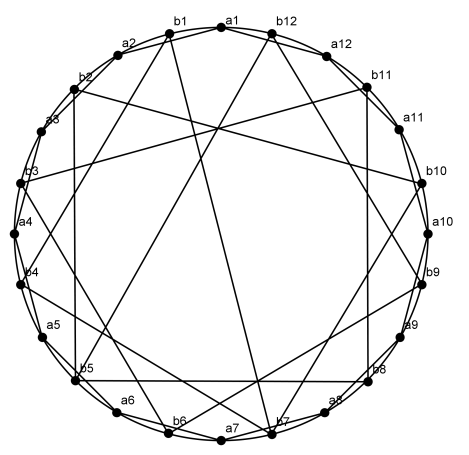

Figure 1. A graph $G$ of order 24 containing no $C_{4}$ with $\delta(G)=4$.

(b) Case $2 m=2 k+1, k \geq 9, k \neq 11$.

In this case, if $k \neq 11$ define the vertex-set and edge-set of $G$ as follows.

$V(G)=\left\{c, a_{1}, a_{2}, \ldots, a_{k}, b_{1}, b_{2}, \ldots, b_{k}\right\}$, and

$E(G)=\left\{a_{i} b_{i} \mid i \in[1, k]\right\} \cup\left\{b_{i} a_{i+1}, a_{i} a_{i+1} \mid i \in[1, k-1]\right\} \cup\left\{b_{i} b_{i+3} \mid i \in\right.$ $[1, k-3]\} \cup\left\{b_{1} b_{k-1}, b_{2} b_{k}, a_{1} b_{k-2}, c a_{1}, c a_{k}, c b_{3}, c b_{k}\right\}$

Note that all indices are calculated in $\bmod k$. It is easy to see that each vertex is adjacent to at least four vertices, so $\delta(G)=4$. Now, we will show that $G \nsupseteq C_{4}$. For a contradiction, suppose $G$ contains a $C_{4}$. Since $k \neq 11$, this $C_{4}$ cannot consist of vertices $b_{i}$ only. Therefore, this $C_{4}$ must contain at least one vertex $a_{i}$. Now, consider the following 4 subcases.

- Subcase 1. $a_{i} b_{i} \in C_{4}$ for some $i$. If $a_{i}, b_{i}$ are the first and second vertices in $C_{4}$ then the possible third and fourth vertices are listed in Table 3. However, there is no vertex 4 is connected to vertex 1 . Therefore, this $C_{4}$ cannot contain an edge $a_{i} b_{i}$, for some $i$.

- Subcase 2. $b_{i} a_{i+1} \in C_{4}$ for some $i$ or $c b_{k} \in C_{4}$. From the above subcase, this $C_{4}$ cannot contain an edge $a_{i} b_{i}$, for some $i$. If $b_{i}$ and $a_{i+1}, c$ are the first and second vertices in $C_{4}$ then the possible third and fourth vertices are presented in Table 4. Again, however, no vertex 4 is connected to vertex 1 . Therefore, $b_{i} a_{i+1}$ or $c b_{k}$ cannot be in $C_{4}$, for some $i$.

- Subcase 3. $a_{i} a_{i+1}, c a_{k}$, or $c a_{1} \in C_{4}$, for some $i$. In this case, the possible vertices of this $C_{4}$ can be seen in Table 5. But, no vertex 4 is adjacent to vertex 1 . Therefore, there is no such $C_{4}$ formed in this case.

- Subcase 4. $b_{1} b_{k-1}, b_{2} b_{k}, a_{1} b_{k-2}, c b_{3}$ or $b_{i} b_{i+3} \in C_{4}$ for some $i$. We can assume that $b_{i}$ is the first vertex of a $C_{4}$. Then, the possible vertex of the $C_{4}$ are presented in Table 6. In this case, it is clear that no $C_{4}$ can be formed. Thus, $C_{4} \nsubseteq G$. 


\begin{tabular}{|c|c|c|c|}
\hline vertex 1 & vertex 2 & vertex 3 & vertex 4 \\
\hline \multirow[t]{29}{*}{$a_{i}$} & $b_{i}$ & $a_{i+1}$ & $b_{i+1}$ \\
\hline & & & $a_{i}(i \leq k-1)$ \\
\hline & & & $c(i+1=k)$ \\
\hline & & & $a_{i+2}(i+1 \leq k-1)$ \\
\hline & & $c(i=k)$ & $b_{3}$ \\
\hline & & & $a_{k}$ \\
\hline & & & $a_{1}$ \\
\hline & & & $b_{k}$ \\
\hline & & $b_{i+3}(1 \leq i \leq k-3)$ & $b_{1}(i=k-4)$ \\
\hline & & & $a_{i+3}$ \\
\hline & & & $a_{i+4}(i+3 \leq k-1)$ \\
\hline & & & $c(i+3=k)$ \\
\hline & & & $b_{i+6}(i+3 \leq k-6)$ \\
\hline & & & $a_{1}(i+2=k-2)$ \\
\hline & & & $b_{2}(i+3=k)$ \\
\hline & & $b_{k-1}(i=1)$ & $a_{k-1}$ \\
\hline & & & $a_{k}$ \\
\hline & & & $b_{k-4}$ \\
\hline & & $b_{k}(i=2)$ & c \\
\hline & & & $a_{k}$ \\
\hline & & & $b_{k-3}$ \\
\hline & & $c(i=3)$ & $a_{1}$ \\
\hline & & & $b_{k}$ \\
\hline & & & $a_{k}$ \\
\hline & & & $b_{k}$ \\
\hline & & $a_{1}(i=k-2)$ & $b_{1}$ \\
\hline & & & $a_{2}$ \\
\hline & & & $\begin{array}{ll}c \\
\end{array}$ \\
\hline & & & $b_{k-2}$ \\
\hline
\end{tabular}

Table 3. List of possible vertices of a $C_{4}$ in Subcase 1. 


\begin{tabular}{|l|l|l|l|}
\hline vertex 1 & vertex 2 & vertex 3 & vertex 4 \\
\hline$b_{i}$ & $a_{i+1}(i \leq k-1)$ & $a_{i+2}(i+1 \leq k-1)$ & $b_{i+1}(i+1 \leq k-1)$ \\
\hline & & & $a_{i+3}(i+2 \leq k-1)$ \\
\hline & & & $c(i+2=k)$ \\
\hline & & & $c(i=k-1)$ \\
\hline & & $c(i+1=k)$ & $b_{k-2}(i=k-1)$ \\
\hline & & & $b_{1}$ \\
\hline & & & $b_{3}$ \\
\hline & & & $a_{k}$ \\
\hline & & $a_{i}$ & $c(i=1)$ \\
\hline & & & $b_{k-2}(i=1)$ \\
\hline & & & $b_{i-2}$ \\
\hline & & $a_{k}$ & $b_{i-2}$ \\
\hline & $c(i=k)$ & & $a_{k-1}$ \\
\hline & & $a_{1}$ & $b_{2-1}$ \\
\hline & & & $b_{k-2}$ \\
\hline & & & $b_{3}$ \\
\hline & & & $b_{6}$ \\
\hline & & & \\
\hline
\end{tabular}

Table 4. List of possible vertices of a $C_{4}$ for Subcase 2.

\begin{tabular}{|l|l|l|l|}
\hline vertex 1 & vertex 2 & vertex 3 & vertex 4 \\
\hline$a_{i}$ & $a_{i+1}(i \leq k-1)$ & $a_{i+2}(i+1 \leq k-1)$ & $a_{i+3}(i+1 \leq k-1)(i+2 \leq k-1)$ \\
\hline & & & $c(i+2=k)$ \\
\hline & & $c(i+1=k)$ & $a_{1}$ \\
\hline & & & $a_{k}$ \\
\hline & & & $b_{3}$ \\
\hline & $c(i=1)$ & $a_{k}$ & $a_{k-1}$ \\
\hline & & $b_{3}$ & $b_{6}$ \\
\hline & $c(i=k)$ & $a_{1}$ & $a_{2}$ \\
\hline & & & $b_{k-2}$ \\
\hline & & $b_{3}$ & $b_{6}$ \\
\hline
\end{tabular}

Table 5. List of possible vertices of a $C_{4}$ in Subcase 3 . 


\begin{tabular}{|l|l|l|l|}
\hline vertex 1 & vertex 2 & vertex 3 & vertex 4 \\
\hline$b_{i}$ & $b_{i+3}$ & $b_{i+6}$ & $b_{i+9}$ \\
\hline & & & $b_{1}(i+6=k-1)$ \\
\hline & & & $b_{1}(i+6=k-1)$ \\
\hline & & & $b_{2}(i+6=k)$ \\
\hline & & $b_{1}(i+3=k-1)$ & $b_{4}$ \\
\hline & & $a_{1}(i+3=k-2)$ & \\
\hline & & $b_{2}(i+3=k)$ & $b_{5}$ \\
\hline & $b_{k-1}(i=1)$ & $b_{k-4}$ & $b_{k-7}$ \\
\hline & $a_{1}(i=k-2)$ & & \\
\hline & $c(i=3)$ & $b_{3}$ & $b_{6}$ \\
\hline & $b_{k}(i=2)$ & $b_{k-3}$ & $b_{k-6}$ \\
\hline
\end{tabular}

Table 6. List of possible vertices of a $C_{4}$ in Subcase 4.

For $k=11$, we construct a graph $G$ containing no $C_{4}$ on 23 vertices with $\delta(G)=4$ as depicted in Figure 2.

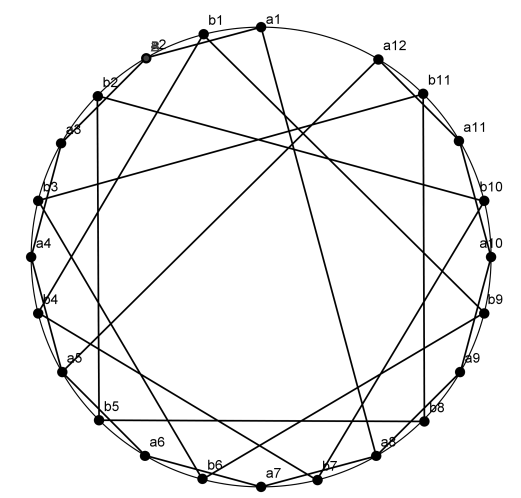

Figure 2. A graph $G$ containing no $C_{4}$ on 23 vertices with $\delta(G)=4$.

(ii) For any even $m \geq 50$, we shall construct a graph $G$ on $m$ vertices with $\delta(G)=5$ and $G \nsupseteq C_{4}$. Let us define the vertex-set and edge-set of $G$ :

$V(G)=\left\{a_{1}, a_{2}, \ldots, a_{m}\right\}$ and

$E(G)=\left\{a_{i} a_{i+1} \mid i \in[1, m]\right\} \cup\left\{a_{i} a_{i+4} \mid i\right.$ odd $\} \cup\left\{a_{i} a_{i+12} \mid i\right.$ even $\}$

$\cup\left\{a_{i} a_{i+8} \mid i=2,4,6,8\right.$, and $i=16 k$, for $\left.k \in[1,\lfloor m / 16\rfloor]\right\}$

$\cup\left\{a_{i} a_{i+16} \mid i=1,3,5, \cdots, 15\right.$, and $i=16 k$, for $\left.k \in[1,\lfloor m / 34\rfloor]\right\}$.

It can be verified easily that $\delta(G)=5$. Now, suppose that $C_{4} \subseteq G$. Let $C_{4}$ be $\left(a_{i_{1}}, a_{i_{2}}, a_{i_{3}}, a_{i_{4}}\right)$ with $i_{2}=i_{1}+x_{1}, i_{3}=i_{2}+x_{2}, i_{4}=i_{3}+x_{3}, i_{1}=i_{4}+x_{4} \bmod m$. Clearly, $G \supseteq C_{4}$ if and only if $m$ divides $x_{1}+x_{2}+x_{3}+x_{4}$. So, $x_{1}+x_{2}+x_{3}+x_{4}=0$ or $x_{1}+x_{2}+x_{3}+x_{4}$ is a multiple of 4 . Observe that the maximum value of $x_{1}+x_{2}+x_{3}+x_{4}=48$ which is achieved when $i$ is even. It is easy to see that $x_{1}+x_{2}+x_{3}+x_{4} \neq 0$. Therefore, $m$ never divides $\left(x_{1}+x_{2}+x_{3}+x_{4}\right)$. Thus, $G \nsupseteq C_{4}$. 
(iii) We will show that $R\left(C_{4}, W_{2 k+1}\right) \leq R\left(C_{4}, W_{2 k}\right)$ for any $k \geq 25$. By Theorem $1.1(i i)$, we have a graph $G$ on $m=2 k+4 \geq 50$ vertices with $\delta(G) \leq 5$ and $G \nsubseteq C_{4}$. Then, $\triangle(\bar{G}) \leq 2 k-2$. Thus, $\bar{G} \nsupseteq W_{2 k}$. Therefore, we obtain that $G$ is a $\left(C_{4}, W_{2 k}, 2 k+4\right)$-good graph. As a consequence, $R\left(C_{4}, W_{2 k}\right) \leq 2 k+5$. Now, let $R\left(C_{4}, W_{2 k}\right)=m$. By Lemma 2.1, there exists a $C_{4}, W_{2 k}, m-1$-good graph $G$ with $\delta(G) \geq m-1-2 k+1=m-2 k$. Thus, $\triangle(\bar{G}) \leq(m-1)-(m-2 k)=2 k-1$. This means that $G$ is also a $\left(C_{4}, W_{2 k+1}, m+1\right)$-good graph. Therefore $R\left(C_{4}, W_{2 k+1}\right) \leq R\left(C_{4}, W_{2 k}\right)$.

(iv) We will show that $R\left(C_{4}, W_{m+n}\right) \geq \max \left\{R\left(C_{4}, W_{m}\right), R\left(C_{4}, W_{n}\right)\right\}$ with $\min \{m, n\} \geq 7$ and $\max \{m, n\} \geq 50$. Without lost of generality, let $R\left(C_{4}, W_{m}\right)=\max \left\{R\left(C_{4}, W_{m}\right), R\left(C_{4}, W_{n}\right)\right\}$. If $m$ is even, by Theorem 1.1(ii) there exists graph $G$ on $m+4$ with $\delta(G)=5$ and $C_{4} \nsubseteq G$. Then, $\triangle(\bar{G}) \leq m-2$. Then, $W_{m} \nsubseteq \bar{G}$. Therefore, we obtain that $G$ is a $\left(C_{4}, W_{m}, m+4\right)$ good graph. As a consequence, $R\left(C_{4}, W_{m}\right) \geq m+5$ and by Theorem 1.1(3), we have $R\left(C_{4}, W_{m}\right) \geq m+5$ for all $m \geq 50$. Now, let $R\left(C_{4}, W_{m}\right)=p$. By Lemma 2.1, there exists a $\left(C_{4}, W_{m}, p-1\right)$-good graph $G$ with $\delta(G) \geq p-1-m+1=p-m$. Thus, $\triangle(\bar{G}) \leq(p-1)-(p-m)=m-1 \leq m+n-1$. This means that $G$ is also a $R\left(C_{4}, W_{m+n}, p-1\right)$-good graph. Therefore, $R\left(C_{4}, W_{m+n}\right) \geq \max \left\{R\left(C_{4}, W_{m}\right)\right.$.

\section{Proof Theorem 1.2.}

(i) We will show that $m+6 \leq R\left(C_{4}, W_{m}\right) \leq m+7$, for $46 \leq m \leq 51$. Hoffman and Singleton [??] have constructed a $(7,5)$-cage $H S_{50}$ as follow. Let $V\left(H S_{50}\right)=\left\{a_{1}, a_{2}, \cdots, a_{50}\right\}$. All edges of $H S_{50}$ are presented in Table 7.

We construct a new graph $G_{i}$ on $i$ vertices, for each $i \in[51,56]$ as follows.

$$
\begin{aligned}
V\left(G_{51}\right)= & V\left(H S_{50}\right) \cup\{51\} \\
E\left(G_{51}\right)= & E\left(H S_{50}\right) \backslash\{(1,2),(2,34),(20,21),(21,22),(19,41),(34,41)\} \\
& \cup\{(51, i) \mid i \in\{1,2,19,21,34,41\}\} \\
V\left(G_{52}\right)= & V\left(G_{51}\right) \cup\{52\} \\
E\left(G_{52}\right)= & E\left(G_{51}\right) \backslash\{(10,11),(11,12),(3,4),(3,16),(5,20)\} \\
& \cup\{(52, i) \mid i \in\{2,3,11,12,16,20\}\}
\end{aligned}
$$




\begin{tabular}{|c|c|c|c|c|c|c|c|c|c|c|c|c|c|c|c|}
\hline 1 & 2 & 19 & 29 & 32 & 44 & 47 & 50 & 26 & 10 & 13 & 22 & 25 & 27 & 33 & 50 \\
\hline 2 & 1 & 3 & 6 & 10 & 21 & 24 & 34 & 27 & 3 & 19 & 26 & 28 & 31 & 39 & 43 \\
\hline 3 & 2 & 4 & 8 & 16 & 27 & 37 & 46 & 28 & 5 & 14 & 23 & 27 & 29 & 34 & 45 \\
\hline 4 & 3 & 5 & 11 & 18 & 22 & 32 & 48 & 29 & 1 & 11 & 17 & 25 & 28 & 30 & 37 \\
\hline 5 & 4 & 6 & 9 & 20 & 28 & 38 & 50 & 30 & 6 & 15 & 22 & 29 & 35 & 46 & 31 \\
\hline 6 & 2 & 5 & 7 & 13 & 30 & 40 & 43 & 31 & 9 & 12 & 24 & 27 & 30 & 32 & 49 \\
\hline 7 & 6 & 8 & 116 & 19 & 23 & 33 & 49 & 32 & 1 & 4 & 14 & 31 & 33 & 36 & 40 \\
\hline 8 & 3 & 7 & 9 & 14 & 25 & 35 & 44 & 33 & 7 & 17 & 26 & 32 & 34 & 38 & 46 \\
\hline 9 & 5 & 8 & 10 & 17 & 31 & 41 & 47 & 34 & 2 & 12 & 28 & 33 & 35 & 41 & 48 \\
\hline 10 & 2 & 9 & 11 & 15 & 26 & 36 & 45 & 35 & 8 & 18 & 30 & 34 & 36 & 39 & 50 \\
\hline 11 & 4 & 7 & 10 & 12 & 29 & 39 & 42 & 36 & 10 & 20 & 23 & 35 & 32 & 37 & 43 \\
\hline 12 & 11 & 13 & 16 & 20 & 31 & 34 & 44 & 37 & 3 & 13 & 29 & 36 & 38 & 41 & 49 \\
\hline 13 & 6 & 12 & 14 & 18 & 26 & 37 & 47 & 38 & 5 & 15 & 24 & 33 & 37 & 39 & 44 \\
\hline 14 & 8 & 13 & 15 & 21 & 28 & 32 & 42 & 39 & 11 & 21 & 27 & 35 & 38 & 40 & 47 \\
\hline 15 & 10 & 14 & 16 & 19 & 30 & 38 & 48 & 40 & 6 & 16 & 25 & 32 & 39 & 41 & 45 \\
\hline 16 & 3 & 12 & 15 & 17 & 23 & 40 & 50 & 41 & 9 & 19 & 22 & 34 & 37 & 40 & 42 \\
\hline 17 & 9 & 16 & 18 & 21 & 29 & 33 & 43 & 42 & 11 & 14 & 24 & 41 & 43 & 46 & 50 \\
\hline 18 & 4 & 13 & 17 & 19 & 24 & 35 & 45 & 43 & 6 & 17 & 27 & 36 & 42 & 44 & 48 \\
\hline 19 & 1 & 7 & 15 & 18 & 20 & 27 & 41 & 44 & 1 & 8 & 12 & 22 & 38 & 43 & 45 \\
\hline 20 & 5 & 12 & 15 & 21 & 25 & 36 & 46 & 45 & 10 & 18 & 28 & 40 & 44 & 46 & 49 \\
\hline 21 & 2 & 14 & 17 & 20 & 22 & 39 & 49 & 46 & 3 & 20 & 30 & 33 & 42 & 45 & 47 \\
\hline 22 & 4 & 21 & 23 & 26 & 30 & 41 & 44 & 47 & 1 & 9 & 13 & 23 & 39 & 46 & 48 \\
\hline 23 & 7 & 16 & 22 & 24 & 28 & 36 & 47 & 48 & 4 & 15 & 25 & 34 & 43 & 47 & 49 \\
\hline 24 & 2 & 18 & 23 & 25 & 31 & 38 & 42 & 49 & 7 & 21 & 31 & 37 & 45 & 48 & 50 \\
\hline 25 & 8 & 20 & 24 & 26 & 29 & 40 & 48 & 50 & 1 & 5 & 16 & 26 & 35 & 42 & 49 \\
\hline
\end{tabular}

Table 7. The Hoffman and Singleton graph $H S_{50}$. 


$$
\begin{aligned}
V\left(G_{53}\right)= & V\left(G_{52}\right) \cup\{53\} \\
E\left(G_{53}\right)= & E\left(G_{52}\right) \backslash\{(5,9),(4,11),(31,32)\} \\
& \cup\{(53, i) \mid i \in\{4,5,9,10,11,, 31\}\} \\
V\left(G_{54}\right)= & V\left(G_{53}\right) \cup\{54\} \\
E\left(G_{54}\right)= & E\left(G_{53}\right) \backslash\{(22,30),(18,35),(30,35),(21,39)\} \\
& \cup\{(54, i) \mid i \in\{4,21,27,30,35,39\}\} \\
V\left(G_{55}\right)= & V\left(G_{54}\right) \cup\{55\} \\
E\left(G_{55}\right)= & E\left(G_{54}\right) \backslash\{(1,50),(5,50),(32,36),(35,36)\} \\
& \cup\{(55, i) \mid i \in\{1,5,19,35,36,50\}\} \\
V\left(G_{56}\right)= & V\left(G_{55}\right) \cup\{56\} \\
E\left(G_{56}\right)= & E\left(G_{55}\right) \backslash\{(7,23),(17,33),(16,23),(26,33)\} \\
& \cup\{(56, i) \mid i \in\{7,16,17,22,23,33\}\}
\end{aligned}
$$

Consider graph $G_{51}$. Clearly, $\delta\left(G_{51}\right)=6$. Now, we will show that $C_{4} \nsubseteq G_{51}$. For a contradiction, suppose $C_{4} \subseteq G_{51}$. If $C_{4} \subseteq G_{51}$ then this $C_{4}$ must consists of vertex 51 , two vertices adjacent to 51, say $x$ and $y$, and one other vertex adjacent to $x$ and $y$. If vertex 51 is the first vertex of this $C_{4}$ then $\{x, y\} \subset\left\{a_{1}, a_{2}, a_{19}, a_{21}, a_{34}, a_{41}\right\}$. However, there is no other vertex adjacent to both $x$ and $y$, see Figure 3 . Therefore, there is no $C_{4}$ in $G_{51}$. Similarly, we have show that $\delta\left(G_{i}\right)=6$ and $C_{4} \nsubseteq G_{i}$ for all $i \in\{52, \cdots, 56\}$.

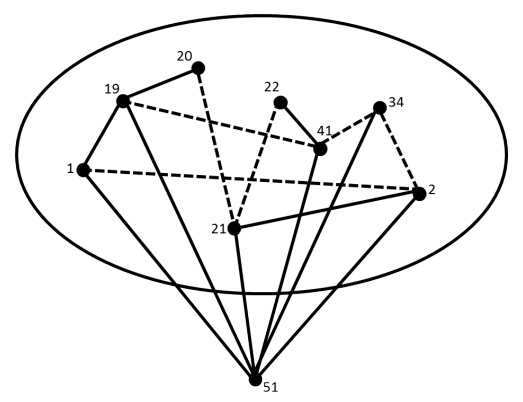

Figure 3. Possible $C_{4}$ in $G_{51}$.

Now, we have $\Delta\left(\bar{G}_{i}\right) \leq i-7$. Thus, $W_{i-5} \nsubseteq \bar{G}_{i}$. As a consequence, $R\left(C_{4}, W_{i-5}\right) \geq i$ for all $i \in\{51, \ldots, 56\}$. By Theorem 2.1, $R\left(C_{4}, W_{m}\right) \leq m+7$, for $46 \leq m \leq 51$. Thus, $m+6 \leq R\left(C_{4}, W_{m}\right) \leq m+7$ for $46 \leq m \leq 51$.

(ii) We will show that $m+8 \leq R\left(C_{4}, W_{m}\right) \leq m+9$ for $79 \leq m \leq 82$. From [2], there exists a $(9,5)$-graph on 96 vertices, call it $G_{96}$. Let $V\left(G_{96}\right)=\{0,1,2, \cdots, 95\}$ and all edges of graph $G_{96}$ are presented in Table 8. We construct a graph $G_{i}$ on $i$ vertices for $86 \leq i \leq 95$, $\delta\left(G_{i}\right)=8$ and $C_{4} \nsubseteq G_{i}$. Graph $G_{i}$ is obtained by removing a single vertex of $G_{i+1}$ as follows:

$$
V\left(G_{i}\right)=V\left(G_{i+1}\right) \backslash\{a\}
$$


with $a$ respectively $95,79,1,13,18,36,40,46,47,63$. Now, we have $\Delta\left(\bar{G}_{i}\right) \leq i-9$. Thus, $W_{i-7} \nsubseteq \bar{G}_{i}$. Therefore, we obtain that $G_{i}$ is $\left(C_{4}, W_{i-7}, i\right)$-good graph. As a consequence $R\left(C_{4}, W_{i-7}\right) \geq i+1$ for $79 \leq m \leq 87$ with $m=i-7$. By Theorem $2.1, R\left(C_{4}, W_{m}\right) \leq$ $m+9$, for $79 \leq m \leq 82$.

(iii) By Theorem 2.1, $R\left(C_{4}, W_{m}\right) \leq m+10$, for $83 \leq m \leq 87$ and by the constructions in Theorem 1.2 (ii), we have $R\left(C_{4}, W_{m}\right) \geq m+8$, for $83 \leq m \leq 87$.

(iv) We will show that $97 \leq R\left(C_{4}, W_{88}\right) \leq 98$ and $m+8 \leq R\left(C_{4}, W_{m}\right) \leq m+10$ for $89 \leq m \leq 93$. Graph $G_{96}$ is $(9,5)$-graph. Thus, $\Delta\left(\bar{G}_{96}\right)=96-1-9=86$. Therefore, we obtain that $G_{96}$ is a $\left(C_{4}, G_{88}, 96\right)$-good graph and $G_{96}$ is a $\left(C_{4}, G_{89}, 96\right)$-good graph. As a consequence $R\left(C_{4}, G_{88}\right) \geq 97$ and $R\left(C_{4}, G_{89}\right) \geq 97$. For $90 \leq m \leq 93$, we construct graph $G_{i}$ on $i$ vertices, with $97 \leq i \leq 100$ as follows.

$$
\begin{aligned}
V\left(G_{97}\right)= & V\left(G_{96}\right) \cup\{96\} \\
E\left(G_{97}\right)= & E\left(G_{96}\right) \backslash\{(8,16),(53,77),(16,93),(0,8),(0,77),(34,82),(5,53),(24,58)\} \\
& \cup\{(96, i) \mid i \in\{0,8,16,24,53,77,93,82\}\} \\
V\left(G_{98}\right)= & V\left(G_{97}\right) \cup\{97\} \\
E\left(G_{98}\right)= & E\left(G_{97}\right) \cup\{(97, i) \mid i \in\{34,26,18,10,58,5,87,64\}\} \\
& \backslash\{(18,26),(10,18),(60,26),(5,64),(10,87),(58,82),(63,87),(9,64),(34,80)\} \\
V\left(G_{99}\right)= & V\left(G_{98}\right) \cup\{98\} \\
E\left(G_{99}\right)= & E\left(G_{98}\right) \cup\{(98, i) \mid i \in\{3,6,11,19,48,65,80,88\}\} \\
& \backslash\{(3,11),(31,65),(11,19),(6,46),(3,52),(6,88),(19,65),(56,80),(72,88), \\
& (14,48)\} \\
V\left(G_{100}\right)= & V\left(G_{99}\right) \cup\{99\} \\
E\left(G_{100}\right)= & E\left(G_{99}\right) \cup\{(99, i) \mid i \in\{1,7,25,33,41,43,52,81\}\} \\
& \backslash\{(33,41),(1,41),(1,50),(43,89),(33,92),(25,54),(7,89),(7,62),(25,84),
\end{aligned}
$$

From the construction, we have $\Delta\left(\bar{G}_{i}\right)=i-9$ for $97 \leq i \leq 100$. Thus, $W_{i-7} \nsubseteq \bar{G}_{i}$. Therefore, we obtain that $G_{i}$ is $\left(C_{4}, W_{i-7}, i\right)$-good graph. As consequence $R\left(C_{4}, W_{m}\right) \geq$ $m+8$ for $90 \leq m \leq 93$ with $m=i-7$. By Theorem 2.1, $R\left(C_{4}, W_{m}\right) \leq m+10$ for $88 \leq m \leq 93$.

\section{Acknowledgment}

This research was supported by Research Grant "Program Riset Unggulan ITB-DIKTI", Ministry of Research, Technology and Higher Education, Indonesia. 


\begin{tabular}{|c|c|c|c|c|c|c|c|c|c|c|c|c|c|c|c|c|c|c|c|}
\hline 0 & 8 & 40 & 48 & 49 & 55 & 59 & 77 & 82 & 94 & 48 & 0 & 2 & 14 & 19 & 37 & 41 & 47 & 64 & 72 \\
\hline 1 & 9 & 41 & 49 & 50 & 56 & 60 & 78 & 83 & 95 & 49 & 0 & 1 & 3 & 15 & 20 & 38 & 42 & 65 & 73 \\
\hline 2 & 10 & 42 & 48 & 50 & 51 & 57 & 61 & 79 & 84 & 50 & 1 & 2 & 4 & 16 & 21 & 39 & 43 & 66 & 74 \\
\hline 3 & 11 & 43 & 49 & 51 & 52 & 58 & 62 & 80 & 85 & 51 & 2 & 3 & 5 & 17 & 22 & 40 & 44 & 67 & 75 \\
\hline 4 & 12 & 44 & 50 & 52 & 53 & 59 & 63 & 81 & 86 & 52 & 3 & 4 & 6 & 18 & 23 & 41 & 45 & 68 & 76 \\
\hline 5 & 13 & 45 & 51 & 53 & 54 & 60 & 64 & 82 & 87 & 53 & 4 & 5 & 7 & 19 & 24 & 42 & 46 & 69 & 77 \\
\hline 6 & 14 & 46 & 52 & 54 & 55 & 61 & 65 & 83 & 88 & 54 & 5 & 6 & 8 & 20 & 25 & 43 & 47 & 70 & 78 \\
\hline 7 & 15 & 47 & 53 & 55 & 56 & 62 & 66 & 84 & 89 & 55 & 0 & 6 & 7 & 9 & 21 & 26 & 44 & 71 & 79 \\
\hline 8 & 0 & 16 & 54 & 56 & 57 & 63 & 67 & 85 & 90 & 56 & 1 & 7 & 8 & 10 & 22 & 27 & 45 & 80 & 88 \\
\hline 9 & 1 & 17 & 55 & 57 & 58 & 64 & 68 & 86 & 91 & 57 & 2 & 8 & 9 & 11 & 23 & 28 & 46 & 81 & 89 \\
\hline 10 & 2 & 18 & 56 & 58 & 59 & 65 & 69 & 87 & 92 & 58 & 3 & 9 & 10 & 12 & 24 & 29 & 47 & 82 & 90 \\
\hline 11 & 3 & 19 & 57 & 59 & 60 & 66 & 70 & 88 & 93 & 59 & 0 & 4 & 10 & 11 & 13 & 25 & 30 & 83 & 91 \\
\hline 12 & 4 & 20 & 58 & 60 & 61 & 67 & 71 & 89 & 94 & 60 & 1 & 5 & 11 & 12 & 14 & 26 & 31 & 84 & 92 \\
\hline 13 & 5 & 21 & 59 & 61 & 62 & 68 & 72 & 90 & 95 & 61 & 2 & 6 & 12 & 13 & 15 & 27 & 32 & 85 & 93 \\
\hline 14 & 6 & 22 & 48 & 60 & 62 & 63 & 69 & 73 & 91 & 62 & 3 & 7 & 13 & 14 & 16 & 28 & 33 & 86 & 94 \\
\hline 15 & 7 & 23 & 49 & 61 & 63 & 64 & 70 & 74 & 92 & 63 & 4 & 8 & 14 & 15 & 17 & 29 & 34 & 87 & 95 \\
\hline 16 & 8 & 24 & 50 & 62 & 64 & 65 & 71 & 75 & 93 & 64 & 5 & 9 & 15 & 16 & 18 & 30 & 35 & 48 & 80 \\
\hline 17 & 9 & 25 & 51 & 63 & 65 & 66 & 72 & 76 & 94 & 65 & 6 & 10 & 16 & 17 & 19 & 31 & 36 & 49 & 81 \\
\hline 18 & 10 & 26 & 52 & 64 & 66 & 67 & 73 & 77 & 95 & 66 & 7 & 11 & 17 & 18 & 20 & 32 & 37 & 50 & 82 \\
\hline 19 & 11 & 27 & 48 & 53 & 65 & 67 & 68 & 74 & 78 & 67 & 8 & 12 & 18 & 19 & 21 & 33 & 38 & 51 & 83 \\
\hline 20 & 12 & 28 & 49 & 54 & 66 & 68 & 69 & 75 & 79 & 68 & 9 & 13 & 19 & 20 & 22 & 34 & 39 & 52 & 84 \\
\hline 21 & 13 & 29 & 50 & 55 & 67 & 69 & 70 & 76 & 80 & 69 & 10 & 14 & 20 & 21 & 23 & 35 & 40 & 53 & 85 \\
\hline 22 & 14 & 30 & 51 & 56 & 68 & 70 & 71 & 77 & 81 & 70 & 11 & 15 & 21 & 22 & 24 & 36 & 41 & 54 & 86 \\
\hline 23 & 15 & 31 & 52 & 57 & 69 & 71 & 72 & 78 & 82 & 71 & 12 & 16 & 22 & 23 & 25 & 37 & 42 & 55 & 87 \\
\hline 24 & 16 & 32 & 53 & 58 & 70 & 72 & 73 & 79 & 83 & 72 & 13 & 17 & 23 & 24 & 26 & 38 & 43 & 48 & 88 \\
\hline 25 & 17 & 33 & 54 & 59 & 71 & 73 & 74 & 80 & 84 & 73 & 14 & 18 & 24 & 25 & 27 & 39 & 44 & 49 & 89 \\
\hline 26 & 18 & 34 & 55 & 60 & 72 & 74 & 75 & 81 & 85 & 74 & 15 & 19 & 25 & 26 & 28 & 40 & 45 & 50 & 90 \\
\hline 27 & 19 & 35 & 56 & 61 & 73 & 75 & 76 & 82 & 86 & 75 & 16 & 20 & 26 & 27 & 29 & 41 & 46 & 51 & 91 \\
\hline 28 & 20 & 36 & 57 & 62 & 74 & 76 & 77 & 83 & 87 & 76 & 17 & 21 & 27 & 28 & 30 & 42 & 47 & 52 & 92 \\
\hline 29 & 21 & 37 & 58 & 63 & 75 & 77 & 78 & 84 & 88 & 77 & 0 & 18 & 22 & 28 & 29 & 31 & 43 & 53 & 93 \\
\hline 30 & 22 & 38 & 59 & 64 & 76 & 78 & 79 & 85 & 89 & 78 & 1 & 19 & 23 & 29 & 30 & 32 & 44 & 54 & 94 \\
\hline 31 & 23 & 39 & 60 & 65 & 77 & 79 & 80 & 86 & 90 & 79 & 2 & 20 & 24 & 30 & 31 & 33 & 45 & 55 & 95 \\
\hline 32 & 24 & 40 & 61 & 66 & 78 & 80 & 81 & 87 & 91 & 80 & 3 & 21 & 25 & 31 & 32 & 34 & 46 & 56 & 64 \\
\hline 33 & 25 & 41 & 62 & 67 & 79 & 81 & 82 & 88 & 92 & 81 & 4 & 22 & 26 & 32 & 33 & 35 & 47 & 57 & 65 \\
\hline 34 & 26 & 42 & 63 & 68 & 80 & 82 & 83 & 89 & 93 & 82 & 0 & 5 & 23 & 27 & 33 & 34 & 36 & 58 & 66 \\
\hline 35 & 27 & 43 & 64 & 69 & 81 & 83 & 84 & 90 & 94 & 83 & 1 & 6 & 24 & 28 & 34 & 35 & 37 & 59 & 67 \\
\hline 36 & 28 & 44 & 65 & 70 & 82 & 84 & 85 & 91 & 95 & 84 & 2 & 7 & 25 & 29 & 35 & 36 & 38 & 60 & 68 \\
\hline 37 & 29 & 45 & 48 & 66 & 71 & 83 & 85 & 86 & 92 & 85 & 3 & 8 & 26 & 30 & 36 & 37 & 39 & 61 & 69 \\
\hline 38 & 30 & 46 & 49 & 67 & 72 & 84 & 86 & 87 & 93 & 86 & 4 & 9 & 27 & 31 & 37 & 38 & 40 & 62 & 70 \\
\hline 39 & 31 & 47 & 50 & 68 & 73 & 85 & 87 & 88 & 94 & 87 & 5 & 10 & 28 & 32 & 38 & 39 & 41 & 63 & 71 \\
\hline 40 & 0 & 32 & 51 & 69 & 74 & 86 & 88 & 89 & 95 & 88 & 6 & 11 & 29 & 33 & 39 & 40 & 42 & 56 & 72 \\
\hline 41 & 1 & 33 & 48 & 52 & 70 & 75 & 87 & 89 & 90 & 89 & 7 & 12 & 30 & 34 & 40 & 41 & 43 & 57 & 73 \\
\hline 42 & 2 & 34 & 49 & 53 & 71 & 76 & 88 & 90 & 91 & 90 & 8 & 13 & 31 & 35 & 41 & 42 & 44 & 58 & 74 \\
\hline 43 & 3 & 35 & 50 & 54 & 72 & 77 & 89 & 91 & 92 & 91 & 9 & 14 & 32 & 36 & 42 & 43 & 45 & 59 & 75 \\
\hline 44 & 4 & 36 & 51 & 55 & 73 & 78 & 90 & 92 & 93 & 92 & 10 & 15 & 33 & 37 & 43 & 44 & 46 & 60 & 76 \\
\hline 45 & 5 & 37 & 52 & 56 & 74 & 79 & 91 & 93 & 94 & 93 & 11 & 16 & 34 & 38 & 44 & 45 & 47 & 61 & 77 \\
\hline 46 & 6 & 38 & 53 & 57 & 75 & 80 & 92 & 94 & 95 & 94 & 0 & 12 & 17 & 35 & 39 & 45 & 46 & 62 & 78 \\
\hline 47 & 7 & 39 & 48 & 54 & 58 & 76 & 81 & 93 & 95 & 95 & 1 & 13 & 18 & 36 & 40 & 46 & & 63 & 79 \\
\hline
\end{tabular}

Table 8. The graph $G_{96}$. 


\section{References}

[1] J. Dybizbański, T. Dzido, On Some Ramsey Numbers for Quadrilaterals versus Wheels, Graphs and Combinatorics 30 (2014), 573-579.

[2] K.K. Tse, On the Ramsey Turán Number for Quadrilateral, Utilitas Mathematica 79 (2003), 51-58.

[3] L.K.Jørgensen, Girth 5 Graphs from Relative Difference Sets, Discreate Mathematics 293 (2005), 177-184.

[4] S.P Radziszowski, Small Ramsey Numbers, Electronic Journal of Combinatorics (2014), DS1.14.

[5] S.P Radziszowski, K.K.Tse, A computational approach for the Ramsey numbers $R\left(C_{4}, K_{n}\right)$, Journal of Combinatorial Mathematics and Combinatorial Computing 42 (2002), 195-207.

[6] W. Yali, S. Yongqi, S.P Radziszowski, Wheel and Star-critical Ramsey Numbers for Quadrilateral, Discrete Applied Mathematics 186 (2015), 260-271.

[7] Y. Zhang, H. Broersma, Y. Chen, A Remark on Star- $C_{4}$ and Wheel- $C_{4}$ Ramsey Numbers, Electronic Journal of Graph Theory and Applications 2(2) (2014), 110-114. 